\title{
Light-induced molecular motion of azobenzene- containing molecules: a random-walk model
}

\author{
B Bellini, J Ackermann, H Klein, Ch Grave, Ph Dumas and V Safarov \\ Centre de Recherche sur les Mécanismes Cristallins et en Nanomatériaux, CNRS UPR 7251, \\ Associated to Université Aix-Marseille II et Université Aix-Marseille III, Faculté des Sciences de \\ Luminy, Case 90113288 Marseille, France
}

\begin{abstract}
Since it was first evidenced in 1995, light-induced mass motion in layers of azobenzene-containing molecules has led to diverging interpretations, and it remains partly unexplained. In this paper, we discuss a light-driven randomwalk model where moving chromophores drag the molecule to which they are grafted. It consists in a diffusion motion of the azobenzene functions where each random step follows an isomerizing absorption. After a summary of the main characteristics of the motion, we present the hypotheses of the model and we show how it suits the experimental observations reported. In the frame of this model, where each azobenzene function is put in motion by light, we assess the distance over which an azobenzene-containing molecule can be dragged. Wealsoestimate the energetic output of this dragging process. Finally, we discuss the microscopic origin of these molecular motors and we compare it to the model of thermal ratchets introduced by Feynman and extensively resorted to in Biology nowadays.
\end{abstract}

In 1995, Rochon et al [1] showed that a thin layer of an azobenzene-containing material subjected to a low-intensity modulated blue/green light pattern is significantly altered: an initially flat film at the nanometre scale can reach a corrugation whose amplitude may be up to fifty per cent of the initial thickness. This suggests that the molecules are put in motion upon illumination. The most commonly used set-up to induce mass motion is an interference device with which a sine modulated intensity pattern is cast on a layer at the micron scale. As a result, the free surface is deformed into a surface relief grating, the periodicity of which is equal to that of the interference pattern.

Figure 1 is an AFM image of a surface relief grating inscribed on a PMMA DR1 layer. Ten years after this light-induced motion was evidenced, its origins remain a confused and divisive 


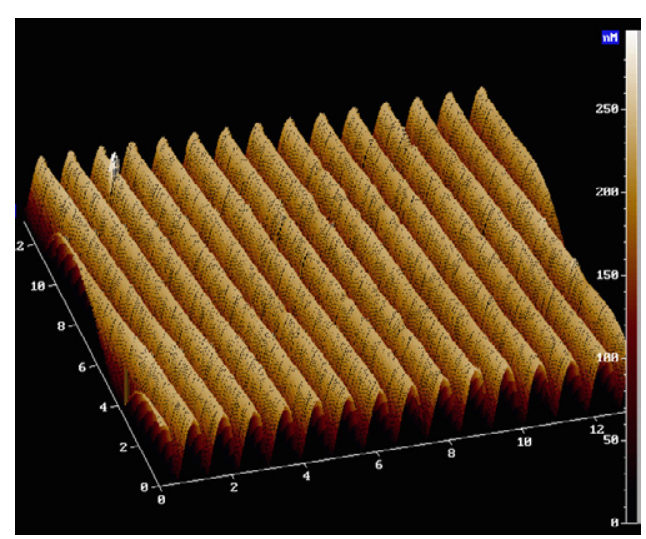

Figure 1. Surface grating inscribed on a PMMA DR1 layer. PMMA DR1 is a polymer which contains azobenzene functions as sides chains.

issue. Several models [2-6] have been developed to account for this phenomenon but all fail to encompass all aspects of it. A major question over this mechanism is to know whether it is a collective or an individual process. Indeed, some view it as a result of interactions between azobenzene moieties while others contend that each azobenzene function is put in motion independently. Beyond its theoretical interest, this question is of major practical importance. Indeed, the possibility of controlling an individual molecule with light opens great perspectives in the context of blooming nanotechnologies. In this paper, we propose an interpretation of this phenomenon in terms of a light-induced individual motion of azobenzene functions. Upon illumination, an azobenzene moiety performs a random-walk motion and drags the polymer to which it is attached. Although still hypothetical, such a molecular locomotive could be a promising tool for the tailoring of molecular architectures, especially for molecular electronics. Following a brief summary of the main experimental features of the light-induced motion, the hypotheses of the random-walk model are presented. Comparing them with the experimental observations allows us to evaluate our model but also to draw geometric and energetic consequences of such an individual motion. In the last section, we discuss the microscopic phenomenon at work and we compare it to the molecular motion based on the thermal ratchet model widely resorted to in Biology.

\section{Experimental facts}

In this section, referring to both the literature and our experimental results, we draw the main characteristics of the light-induced alterations. Then, more specifically, we stick to the growth dynamics of the surface relief gratings presented above. Detailed presentations of these phenomena may be found in two thorough reviews, one written by Delaire and Nakatani [7] in 2002, and the other by Natanshon and Rochon [8] in 2003 on the physical properties of azobenzene-containing compounds. All the experiments presented below were carried out on layers of azobenzene-containing molecules. The choice of layers as an environment to evidence the light-induced molecular motion may appear paradoxical in a work which presents an interpretation of this phenomenon in terms of an individual motion. However, the study of isolated molecules is quite tricky to achieve. Indeed, preparing samples of isolated azobenzenecontaining molecules, referred to as 'sub-monolayers' deposited on a substrate, is a difficult task. Indeed, if one wants to observe the motion of single molecules, it is mandatory to find 
the appropriate substrate which interacts enough with the molecules to allow for a frictiongenerated motion which requires a coupling. But the interaction should not be too strong for the molecule not to remain stuck to the substrate. The search for a procedure for the preparation of 'sub-monolayers' and the attempt at observing an individual molecular motion were undertaken in our research team but they have not led to the observation of moving isolated azobenzenecontaining molecules yet.

\subsection{The main characteristics of the motion}

1.1.1. The phenomenon cannot be reduced to a purely thermal effect. First, it is essential to stress that the phenomenon tackled here is not a purely thermal effect. Indeed, since an absorption process is involved, one could argue that the reported surface alterations result from a plasticization of the film. The increase in temperature at the very surface of the layer is estimated by solving the diffusive heat equation in the film regarded as a semi-infinite plane of initially uniform temperature $T_{0}$ and the surface of which is exposed to a constant light intensity $I_{0}$. In all calculations that follow, the value we take for the intensity of light $I_{0}$ corresponds to our experimental set-up where a $15 \mathrm{~mW}$ beam roughly covers an area of $10 \mathrm{~mm}^{2}$. From the analytical solution for a semi-finite plane, one can drive the expression of the temperature increase versus time at the very surface of the layer:

$$
T(0, t)-T_{0}=\frac{2 I_{0}}{\kappa} \sqrt{\frac{\kappa}{\pi \rho c} t} .
$$

Thus, we can assess the temperature increase at the surface of the film for intensity $I_{0}$. Besides:

$\rho=1.19 \times 10^{3} \mathrm{~kg} \mathrm{~m}^{-3} ; \quad c_{p}=1.46 \times 10^{3} \mathrm{~J} \mathrm{~kg}^{-1} ; \quad \kappa=200 \mathrm{~W} \mathrm{~K}^{-1}$.

After a $1 \mathrm{~h}$ exposure in these conditions the temperature reached by the exposed surface is $6 \mathrm{~K}$, which means that the glass temperature of the polymer, which is around $373 \mathrm{~K}$, is far from being reached. Besides, it does not take more than $10 \mathrm{~min}$ for the grating to be formed.

1.1.2. Influence of the light intensity gradient on the mass motion. At low intensities, such as those referred to here, it has been extensively shown that matter is flowing from bright areas to darker ones. This is clearly evidenced by exposing a PMMA DR1 layer for 5 min to a $15 \mathrm{~mW} / 10 \mathrm{~mm}^{2}$ intensity laser spot. The AFM image in figure 2 shows that matter has moved from the centre of the laser spot, where the intensity of light is the highest, to the less exposed peripheral areas. The migration of matter towards dark areas was first reported by Kumar et al [4] by comparing the deformation resulting from a laser spot with various polarization directions. They also shone on a layer a Fresnel half screen diffraction pattern which, not being symmetrical, unlike the interference pattern usually resorted to, enabled them to tell that matter indeed piles up where the intensity of light is at a minimum.

1.1.3. Influence of the light polarization direction on the mass motion. Another point that stresses the originality of the mass motion process reported in this paper is brought about by the role of the polarization of light. As depicted in figure 2, the laser spot which is shone on the PMMA DR1 layer is linearly polarized along the horizontal direction of the picture. Besides, the AFM image displays an accumulation of matter along that direction on both sides of the hole. This suggests that matter was pushed away from bright areas along the polarization direction. The importance of the polarization state is also evidenced by its effect on the grating's building efficiency. Indeed, given a sinusoidal modulated pattern of one micron spaced fringes, whereas a polarization direction perpendicular to the fringes leads to a significant surface relief 


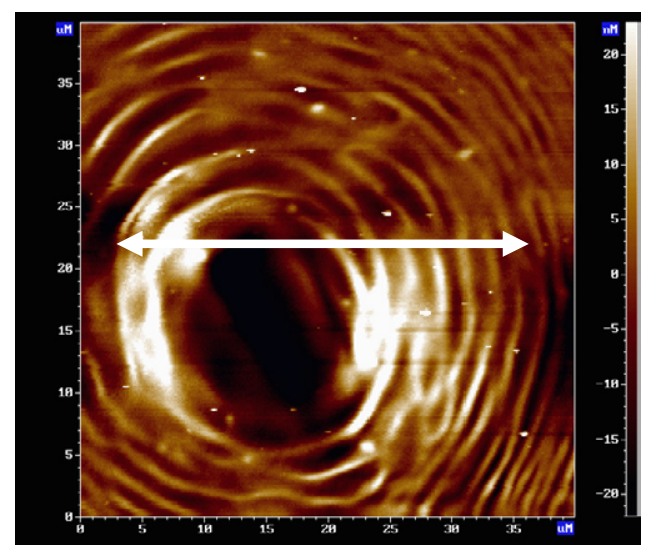

Figure 2. AFM image of a $275 \mathrm{~nm}$ thick PMMA DR1 film after a 10 min backside exposure to a $15 \mathrm{~mW} / 10 \mathrm{~mm}^{2}$ laser beam linearly polarized along the direction indicated by the white arrow above.

grating, a polarization parallel to the fringes does not induce any noteworthy alteration of the layer. Even more striking, an interference pattern of uniform intensity whose only modulation consists in a periodic rotation of the linear polarization direction leads to a significant surface relief grating. Matter is put in motion by the polarization gradient (see figure 3).

1.1.4. A bulk phenomenon. Some view light-induced deformations of layers as a result of the plasticization of their free surface. However, submitting a film to a laser light either shining directly on the free surface or by the backside, through the glass substrate on which the layer is deposited, leads to a similar alteration of the free surface. This strongly suggests that not only does the surface take part in the mass motion process but the whole bulk as well.

1.1.5. Influence of the type of azobenzene-containing molecules. Most light-induced motion experiments have been carried out on azobenzene-containing polymers. In most cases, the azobenzene function is grafted to the backbone as a side chain. In all cases, a mass motion is reported after exposure to a laser pattern (see figure 4). In all these experiments where a polymer is involved, this light-induced motion could be regarded as a consequence of the orientation of the polymers' main chain.

In order to overrule this assumption, we have turned to non-polymeric azobenzenecontaining materials where no main chain may introduce a spurious effect. Among these molecules where no chain is involved, we can mention POPAM dendrimers and Frechet's dendrons. POPAM MO is an arborescence whose periphery consists in methyl orange functions which are bi-substituted azobenzenes (see figure 5).

The group of Professor Vogtle [9] published an article evidencing the formation of a surface relief grating upon illumination of azobenzene-containing dendrimer films. We exposed layers of generation 2, 3 and 4 POPAM MO dendrimers as well as Frechet's dendrons to a modulated intensity pattern similar to the one shone on PMMA DR1. As a result, we obtained surface relief gratings similar to those observed for PMMA layers (see figure 6). Figures 7 and 8 present the surface corrugation observed on the AFM image of a G2MO layer exposed for $5 \mathrm{~min}$ to an interference pattern. The intensity is equal to $15 \mathrm{~mW} / 10 \mathrm{~mm}^{2}$. Another essential characteristic of the process is that no mass motion is observed for non-substituted azobenzene 

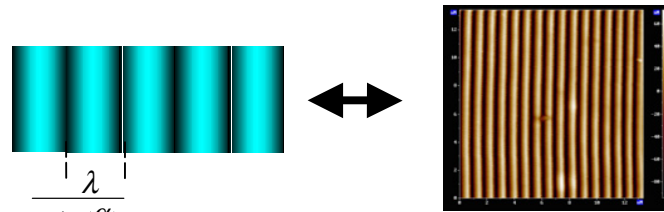

Surface grating the period

$$
\frac{\lambda}{2 \sin \left(\frac{\alpha}{2}\right)}
$$
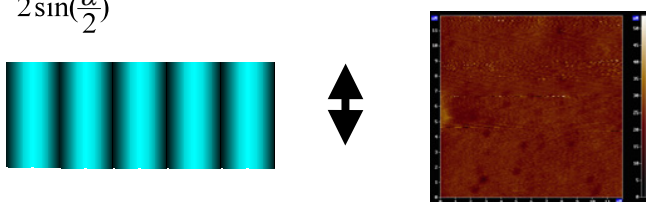

of which is $\frac{\lambda}{2 \sin \left(\frac{\alpha}{2}\right)}$

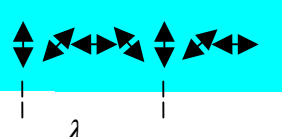

$2 \sin \left(\frac{\alpha}{2}\right)$

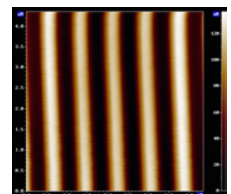

The free surface remains unaltered

Figure 3. Role of the direction of light polarization. In the first two cases, the intensity of light is modulated and the polarization direction is uniform, as indicated by the dark arrow. In the last case, the intensity of light is uniform and the direction of polarization varies in space, as indicated. The resulting deformation is given by the AFM images on the right.<smiles>CCN(CCOC(=O)C(C)(C)C1(C)CCCC1(C)C(C)(C)C(=O)OC)c1ccc(N=Nc2ccc([N+](=O)[O-])cc2)cc1</smiles>

Figure 4. Statistical unit of a PMMA DR1 molecule. The azobenzene function is grafted to the backbone as a side-chain.

functions. A layer of such molecules is not altered upon illumination. In the classification achieved by Rau [10], such bi-substituted functions are referred to as 'pseudostilbenes' or 'push-pull' systems. The absorption bands of the trans to cis or to cis to trans transitions are practically superimposed. So a light of wavelength around 400-500 $\mathrm{nm}$ that activates the trans-cis isomerization also activates the back cis-trans isomerization. The thermal back isomerization is also much faster for pseudostilbenes. The resulting successive trans-cis-trans isomerization cycles are assumed to play a major role in the mass motion process [8]. 


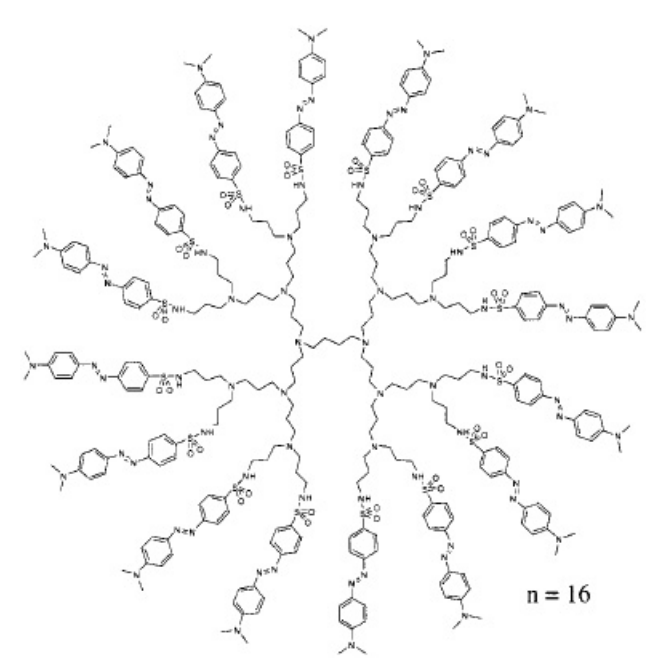

Figure 5. POPAM methyl orange is a third-generation dendrimer with peripheral azobenzenes.
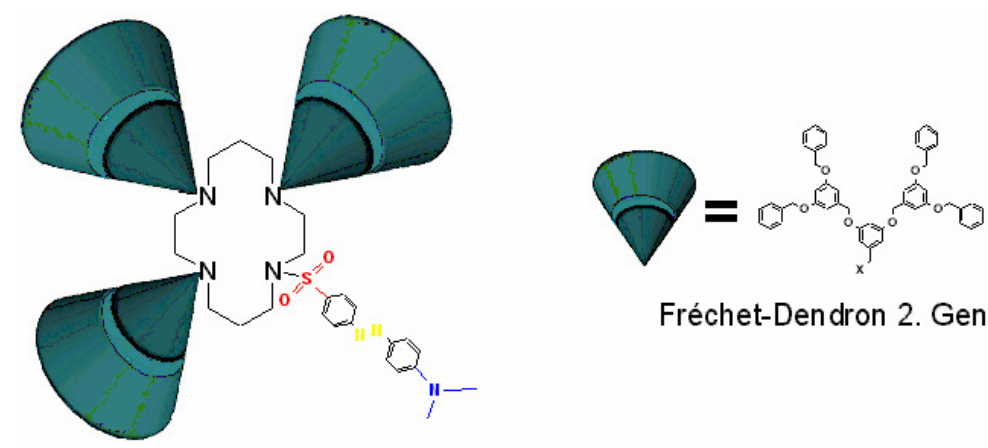

Fréchet-Dendron 2. Generation

Figure 6. Frechet's azo-molecule consists of three dendrons and one azobenzene.

1.1.6. A long-lasting deformation. The AFM images show that, for samples simply stored away from dust, the surface deformation remains unaltered after several years. Thus, the surface tension which tends to flatten the surface plays a minor role in the process and will not be taken into account in the model.

\subsection{The dynamics of the mass motion}

1.2.1. General characteristics of the growth dynamics. In order to follow the light-induced motion in time, we study the evolution of the amplitude of the surface relief grating obtained when layers are exposed to an intensity-modulated interference pattern with a polarization perpendicular to the fringes: $I(x)=2 I_{0}(1+v \cos K x)$. As a criterion of the grating's formation, we have retained the absolute value of the amplitude $h_{1}(x, t)$ which is equal to half of the peak-to-peak amplitude and which is linked to the local thickness of the layer $h(x, t)$ by $h(x, t)=h_{0}+h_{1}(t) \cos K x, h_{0}$ being the initial thickness of the film. The evolution of $h(x, t)$ was obtained with an AFM either by measuring after each exposure of given duration 


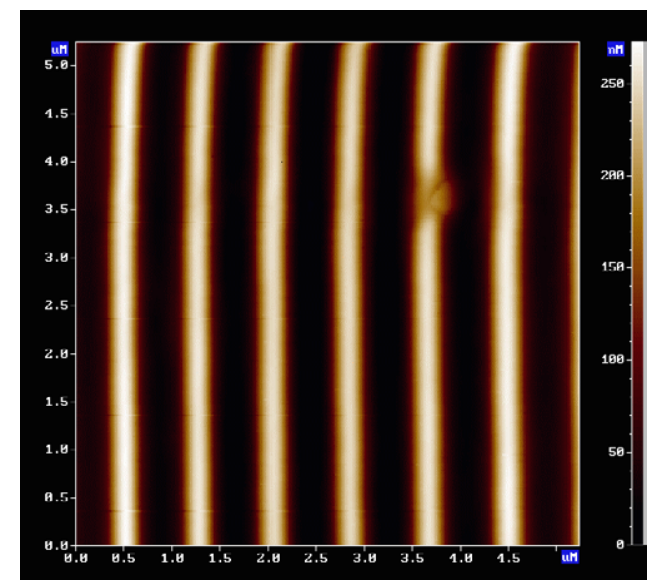

Figure 7. Surface grating obtained on a G2MO layer after a $5 \mathrm{~min}$ exposure to a $15 \mathrm{~mW} / 10 \mathrm{~mm}^{2}$ intensity pattern.

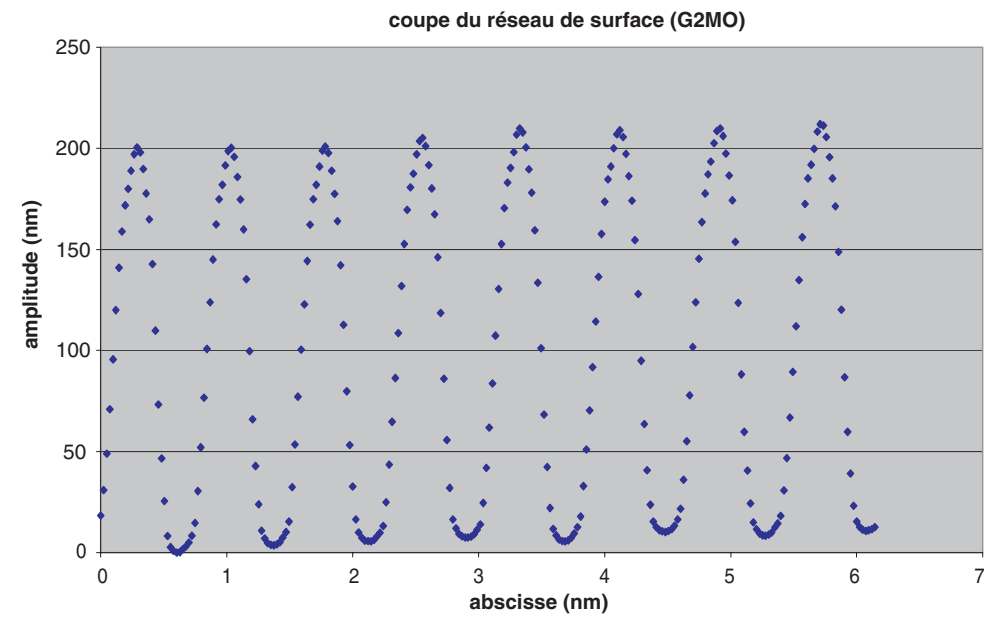

Figure 8. Section evidencing a noteworthy mass motion in G2MO layers. The peak-to-peak amplitude reaches $200 \mathrm{~nm}$.

its effect on the sample or directly by scanning the free surface of the film while the exposure is performed through the backside. In all cases, the dynamics display two parameters.

- A characteristic growth time. In what follows, we shall retain the growth rate at $t=0$ to develop a quantitative analysis since it better suits the calculations computed below.

- A saturation of the amplitude of the grating. This saturation is reached before the layer is altogether dug down to the substrate since the amplitude at saturation is always inferior to the initial thickness of the sample. The origin of this saturation will be discussed in section 3.2.2 (see figure 9).

1.2.2. Influence of the intensity of light. The initial growth rate is proportional to the average intensity of the pattern to which it is subjected. The saturation amplitude is affected as well 


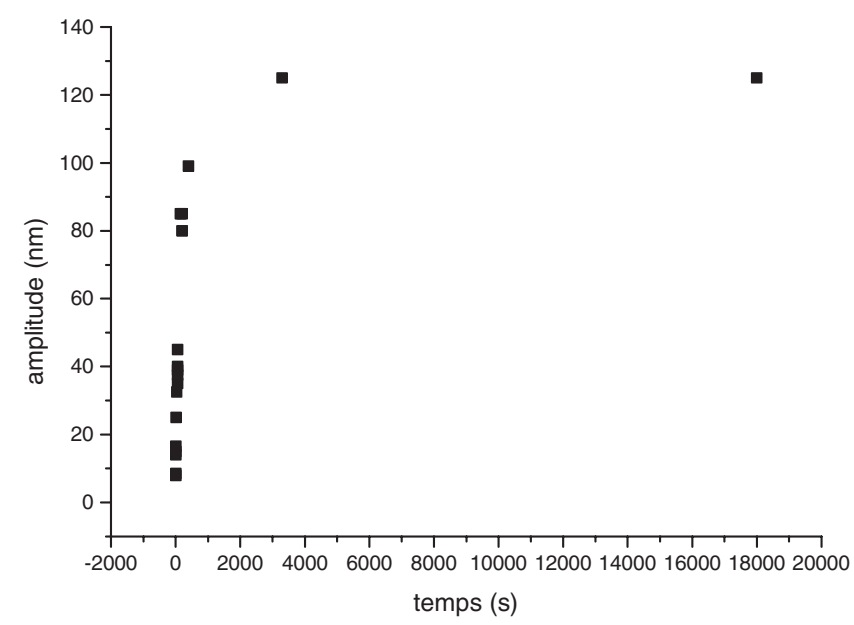

Figure 9. Example of evolution of the amplitude of the surface relief grating versus time.

by the intensity. The table below gives the example of a PMMA DR1 layer of initial thickness $h_{0}=275 \mathrm{~nm}$.

\begin{tabular}{lll}
\hline & $I_{0}=15 \mathrm{~mW} / 10 \mathrm{~mm}^{2}$ & $I_{0}=1.5 \mathrm{~mW} / 10 \mathrm{~mm}^{2}$ \\
\hline Initial growth rate & $1 \pm 0.1 \mathrm{~nm} \mathrm{~s}^{-1}$ & $0.11 \pm 0.02 \mathrm{~nm} \mathrm{~s}^{-1}$ \\
Saturation amplitude & $120 \pm 7 \mathrm{~nm}$ & $80 \pm 5 \mathrm{~nm}$ \\
\hline
\end{tabular}

1.2.3. Influence of the thickness of the layer. The initial thickness of the layer influences the growth rate of the amplitude in a sub-linear way.

\begin{tabular}{lll}
\hline & $h_{0}=275 \mathrm{~nm}$ & $h_{0}=55 \mathrm{~nm}$ \\
\hline Initial growth rate & $1 \pm 0.1 \mathrm{~nm} \mathrm{~s}^{-1}$ & $0.3 \pm 0.05 \mathrm{~nm} \mathrm{~s}^{-1}$ \\
Saturation amplitude & $120 \pm 7 \mathrm{~nm}$ & $30 \pm 4 \mathrm{~nm}$ \\
\hline
\end{tabular}

1.2.4. Influence of the nature of the azobenzene-containing molecule. The nature of the azobenzene-containing compound from which the film is made plays a major role on the characteristic growth time of the film. We study the initial growth rate for various films of similar absorption factor (around 90\%) and subjected to an interference pattern of intensity $I_{0}=15 \mathrm{~mW} / 10 \mathrm{~mm}^{2}$. To get a hint as to how efficient the molecular motion is, we report in the table below the nature of the azobenzene-containing molecule, the initial growth rate and the mass of the statistical unit associated to one azobenzene function. The mass is given in equivalent carbon atoms. Further developments on the light-induced behaviour of various materials will be found in an article to be published by Ackermann et al [11].

\begin{tabular}{llll}
\hline Azobenzene-containing molecule & PMMA DR1 & G2MO & Frechet's \\
\hline Initial growth rate & $1 \mathrm{~nm} \mathrm{~s}^{-1}$ & $2.5 \mathrm{~nm} \mathrm{~s}^{-1}$ & $1 \mathrm{~nm} \mathrm{~s}^{-1}$ \\
$\begin{array}{l}\text { Mass dragged by one AZO function } \\
\text { (equivalent number of carbon atoms) }\end{array}$ & $61 \mathrm{C}$ & $31 \mathrm{C}$ & $155 \mathrm{C}$ \\
\hline
\end{tabular}




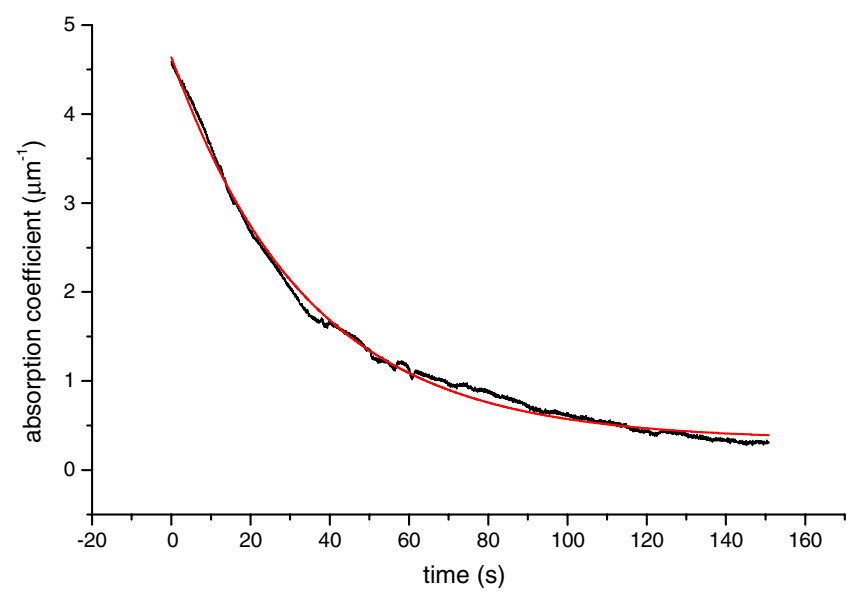

Figure 10. Time evolution of the absorption coefficient $\beta(t)$.

For azobenzene-containing dendrimer films such as G2MO, the formation of the surface relief grating is much faster than for PMMA DR1 layers. Given the last row of the table, we attribute this greater efficiency to the lesser mass dragged by each azobenzene function. The entanglements in the polymer may also account for its lower mobility. More striking, Frechet's molecules layers are put in motion as efficiently as PMMA DR1 ones, whereas the mass associated to one azobenzene function is more than twice as important in this case. One explanation could be that Frechet's molecules are asymmetrical and thus no antagonist solicitations are likely to slow down the motion.

\subsection{Decrease of the optical activity of an azobenzene layer exposed to a laser light}

The transmittance of a layer of azobenzene-containing molecules increases progressively as it is exposed to a laser light. For instance, for a $275 \mathrm{~nm}$ thick PMMA DR1 layer exposed to a laser beam of intensity $I_{0}=15 \mathrm{~mW} / 10 \mathrm{~mm}^{2}$, the transmittance increases first from $30 \%$ to $50 \%$ in a matter of $2 \mathrm{~s}$. Then, it increases at a much lower rate, and reaches $95 \%$ after roughly $40 \mathrm{~min}$. This indicates that azobenzene molecules lose their optical activity and thus progressively fewer and fewer take part in the mass motion process. The fast optical activity loss is reversible. We attribute it to a photo-balance between trans and cis forms. The slow optical activity loss is irreversible. The area on the sample that was exposed loses its colour. These observations suggest that the molecules are bleached by the laser. Thus, the absorption factor $\beta$ of the layer should be regarded as time-dependent. Figure 10 gives the evolution of $\beta(t)$ at the very beginning of the exposure deduced from the increase of its transmission properties.

\section{Hypotheses and parameters of the model}

\subsection{Hypotheses of the model}

The model presented here is a light-driven random walk as underlined by assumption (A3). In this framework, as detailed below, every time a chromophore undergoes an isomerization cycle upon absorption, it performs a random motion of step 1 along the polarization direction one way or another. Thus, it performs a one-dimensional random walk. 
(A1) When an azobenzene function absorbs a photon, it can undergo, given some quantum efficiency, an isomerization from the stable trans form to the cis excited form. In a pseudostilbene environment, as it is the case for the materials that we studied, the back isomerization from the cis to the trans state is activated by the same wavelength so that the azobenzene functions perform trans-cis-trans cycles. The back thermal isomerization is also quite fast (less than one second). Each isomerization goes along with a motion of the azobenzene of a distance $l$.

(A2) This motion of the chromophore tends to drag the molecule to which it is grafted (polymer chain or dendrimer). We consider in the calculation which follows that the volume $V$ dragged by an azobenzene function corresponds to the PMMA DR1 statistical unit to which it belongs or to the fraction of dendrimer to which it is grafted.

For PMMA DR1, we evaluate $V=1 \mathrm{~nm}^{3}$.

(A3) The chromophore's shift of $l$ occurs along the polarization direction. It can go either way with the same probability, which means that it undergoes a one-dimensional random walk.

(A4) We assume that the azobenzene functions grafted to a given azobenzene-containing molecule are not simultaneously optically activated. Otherwise, simultaneous activations would lead to competing motions since each chromophore is assumed to be equally likely to go one way or other along the polarization direction. The resulting motion of such a 'tug-of-war' would be nil. Indeed, as we shall estimate in section 4, an azobenzene dye is activated every second, which is quite a long time compared to the duration of a trans-cis or cis-trans isomerization.

(A5) As mentioned in section 1.3, the optical activity of the azobenzene-containg layers decreases when the layers are subjected to light. In the model, the absorption coefficient is a decreasing function of time in order to account for the decreasing amount of molecules which takes part in the motion.

\subsection{Parameters of the model}

Assumption (A1) enables us to express the number of molecules $n_{\mathrm{d}}$, per unit volume and per unit time, that are isomerized and thus shifted by $l$. It is a fraction $\Phi$ of the molecules that absorb a photon. This fraction corresponds to the sum of the quantum efficiencies of the trans to $c i$ and the cis to trans isomerization process. The expression of the amount of azobenzene $n_{\mathrm{d}}$ put in motion per unit volume and unit time can be found by writing the light intensity decrease undergone by a beam going through a layer of infinitesimal thickness and whose absorption coefficient is $\beta$.

$$
n_{\mathrm{d}}=\phi \beta \frac{\lambda}{h c} I
$$

$\lambda$ is the wavelength of the incident light, $h$ is Planck's constant and $c$ stands for the speed of light. This means that the amount of molecules $n_{\mathrm{d}}$ which are put in motion is proportional to the local intensity. The quantum efficiency of the process $\Phi$ takes into account the quantum yields of trans to cis and cis to trans isomerizations. Following Rau [10], we take $\Phi_{\text {trans-cis }}=0.1$ and $\Phi_{\text {cis-trans }}=0.5$ for PMMA DR1, which belongs to the pseudostilbene category. Assuming that all molecules performing trans-cis or cis-trans isomerization cycles undergo diffusion motion, the resulting quantum efficiency is roughly $\Phi=0,6$.

So, molecular motions are more likely to occur in areas exposed to high intensities. As a consequence of this illumination gradient, a flux of matter is generated from highly exposed 
areas-which are progressively depleted - to darker ones, where the probability of moving is much smaller.

\section{Application of the model to thin films and consequences}

\subsection{Application of the hypotheses: time evolution of the light-induced surface deformation}

In order to compare our model to the experimental facts presented in section 1, it is mandatory to bridge the gap between our 'individual motion' model and the wealth of experimental results concerning layers, that is to say environments with a large number of molecules. To do so, we apply assumptions (A1)-(A4) to an azobenzene-containing material layer deposited on a glass substrate which we fix at $z=0$. Assumption (A5) is overlooked at this point. The initial thickness is assumed to be uniform all over the layer. It is equal to $h_{0}$ along the $z$ direction. The calculation presented below is computed in the case of a frontside exposed layer. Nevertheless, carrying out the calculation for a backside exposed layer would lead to the same expression. This layer is subjected to a light pattern, the intensity of which is modulated along the $x$ axis, and is exponentially attenuated as light is passing through the layer.

- For a layer that is exposed frontside to the light pattern, that is to say directly exposed and for which the maximum intensity is located at is free surface $z=h$, the light intensity profile writes:

$$
I(x, z)=I(x) \mathrm{e}^{-\beta(h-z)}
$$

where $I(x)=I_{0}(1+v \cos K x)$ for two interfering beams polarized perpendicularly to the resulting interference fringes (figure 11). We derive the evolution equation followed by the position $h(x, t)$ along the $z$ axis of the free surface by writing:

* firstly, the expression for the algebraic flux of molecules $J(x, t)$ from left to right, along the $x$ direction, that crosses the surface of matter located at $x$;

* secondly, the conservation of matter for a slice of arbitrary thickness $\mathrm{d} x$.

- The flux $J(x, t)$ is equal to half of the molecules activated in the portion of the layer comprised between $(x-l)$ and $x$, located on the left of the surface minus half of the molecules activated in the portion of the layer between $x$ and $(x+l)$, on the right. The molecules which undergo a shift beyond these slices do not reach the surface located at $x$, given that $l$ is the step of the shift as can be seen in figure 12. Thus, the flux $J(x, t)$ through the surface of matter located at $x$, the length and the width of which are respectively equal to $h(x, t)$ along the $z$ axis and unitary along the $y$ axis, is

$$
\begin{gathered}
J(x, t)=1 / 2 \text { molecule activated between }(x-l) \quad \text { and } \\
x-1 / 2 \text { molecule activated between } x \text { and }(x+l) \\
J(x, t)=\frac{1}{2} \int_{0}^{1} \mathrm{~d} y \int_{x-l}^{x} \mathrm{~d} X \int_{0}^{h(X, t)} \mathrm{d} z \cdot n_{v}(X, z, t) \\
-\frac{1}{2} \int_{0}^{1} \mathrm{~d} y \int_{x}^{x+l} \mathrm{~d} X \int_{0}^{h(X, t)} \mathrm{d} z \cdot n_{v}(X, z, t) .
\end{gathered}
$$

Integrating over $y$, assuming that the shift $l$ of the molecules is small compared to the characteristic distance of the variations of intensity along the $x$ axis and using the expression of $n_{v}$ given above, we get

$$
J(x, t)=-l^{2} \phi \frac{\lambda}{h c} I_{0} \frac{\partial}{\partial x}\left[(1+v \cos (K x))\left(1-\mathrm{e}^{-\beta h(x, t)}\right)\right] .
$$




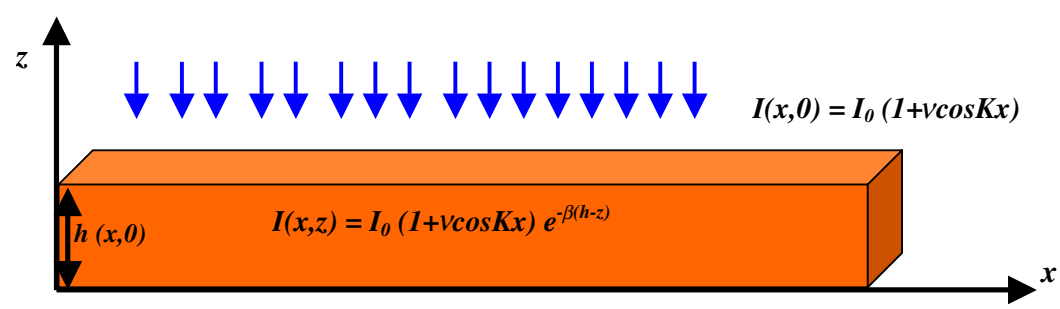

Figure 11. Configuration for a frontside exposed layer.

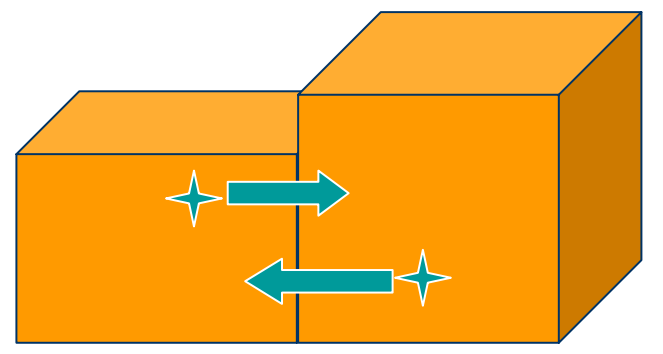

Figure 12. Determination of the molecular flux $J(x, t)$ through the surface located at $x . J(x, t)$ is equal to half of the molecules activated in the volume comprised between $(x-l)$ and $x$ minus half of the molecules activated in the volume comprised between $(x-l)$ and $x$.

- At this point, we can write the law of conservation of matter for a slice of arbitrary thickness $\mathrm{d} x$, whose height and width are respectively equal to $h(x, t)$ along $z$ and unitary along the $y$ axis.

Assuming the density of polymer to remain constant as the morphological changes occur, we can write the volume conservation between time $t$ and time $t+\mathrm{d} t: J(x, t)$ being the flux of chromophores, we need to multiply it by the volume $V$ dragged by each chromophore

$$
1 \mathrm{~d} x[h(x, t+\mathrm{d} t)-h(x, t)]=V J(x, t) \mathrm{d} t-V J(x+\mathrm{d} x, t) \mathrm{d} t .
$$

Using the expression of $J(x, t)$ that we got above, we can write

$$
\frac{\partial h}{\partial t}=l^{2} \phi \frac{\lambda}{h c} I_{0} V \frac{\partial^{2}}{\partial x^{2}}\left[(1+v \cos (K x))\left(1-\mathrm{e}^{-\beta h(x, t)}\right)\right] .
$$

In what follows, we write $D=l^{2} \phi \frac{\lambda}{h c} I_{0} V$.

\subsection{Consequences of the evolution equation}

3.2.1. Expected initial growth rate of the surface grating. As displayed by AFM images, at the beginning of its formation the grating has a sinusoidal shape. Besides, the periodicity of the grating is equal to the periodicity of the interference pattern.

So, in these conditions, we can write $h(x, t)$ during the first seconds as

$$
h(x, t)=h_{0}+h_{1}(t) \cos (K x)+\varepsilon(x, t)
$$

where $\varepsilon(x, t) \ll h_{1}(t) \cos (K x)$.

We can introduce this expression of $h(x, t)$ in the evolution equation and linearize $\mathrm{e}^{-\beta h 1(t)}$ since the AFM images also show that the modulation amplitude of the grating in the 
first seconds (a few nanometres) is small compared to the penetration depth $\frac{1}{\beta}$ (absorption measurements give $120 \mathrm{~nm}$ ):

$\frac{\mathrm{d} h_{1}}{\mathrm{~d} t} \cos (K x)+\frac{\partial \varepsilon}{\partial t}=D \frac{\partial^{2}}{\partial x^{2}}\left((1+v \cos (K x))\left(1-\mathrm{e}^{-\beta h 0}\left(1-\beta h_{1}(t) \cos (K x)+\varepsilon\right)\right)\right)$.

An identification of the terms associated to $\cos (K x)$ gives

$$
\frac{\mathrm{d} h_{1}}{\mathrm{~d} t}(t)=-K^{2} D\left(\mathrm{e}^{-\beta h_{0}} \beta h_{1}(t)+v\left(1-\mathrm{e}^{-\beta h_{0}}\right)\right) .
$$

However tempting it may be to solve this differential equation, one has to keep in mind that its validity is restricted to short times and cannot be extend to derive an expression of $h_{1}(t)$ for any time or of the saturation value.

At $t=0$, the equation gives the expression of the grating's formation rate:

$$
\frac{\mathrm{d} h_{1}}{\mathrm{~d} t}(0)=-K^{2} D \nu A
$$

where $A=\left(1-\mathrm{e}^{-\beta h 0}\right)$ is the absorption factor of the initially flat film of thickness $h_{0}$. We note that $\frac{\mathrm{d} h_{1}}{\mathrm{~d} t}(0)<0$, which is in accordance with the fact that the layer is depleted in areas where the intensity of light is maximal. Moreover, this expression enables us to compare the initial growth rate of two films differing in the nature of the azobenzene-containing molecule without knowing the absorption coefficient $\beta$ and the thickness $h_{0}$ of the films. One just needs to know the fraction $A$ of the light intensity of wavelength $473 \mathrm{~nm}$ that the film initially absorbs. This expression derived from the model assumed in section 2.1 is in accordance with the experimental results presented in section 1.2.2.

- As the experimental initial growth rate, it is proportional to the intensity through the medium of $D$.

- It also accounts for the sub-linear effect of the initial thickness $h_{0}$.

3.2.2. Origin of the saturation: more than just the diffusion to it. Besides the bleaching process, the light-driven diffusion model could in itself lead to a saturation of the surface relief grating growth. Indeed, one can figure out a situation such that motions in dark and highly populated areas are exactly compensated by motions in depleted zones where a higher light intensity induces more excitations of the chromophores.

However, if we do not take the bleaching process into account, that is if we set $\beta$ as a constant $\beta_{0}$, the evolution equation of the free surface $h(x, t)$ admits a saturation only in marginal cases. Indeed, if we assume that the grating amplitude reaches a saturation value $\frac{\partial h}{\partial t}=0$, the evolution equation leads to a free surface profile which can be written as

$$
h_{\mathrm{SAT}}(x) \frac{1}{\beta} \ln \left(\frac{1+v \cos K x}{(1-C)+v \cos K x}\right)
$$

where $v \approx 0.85$ and thickness such that $h_{0}>55 \mathrm{~nm}$ and $C$ is a constant of integration. In order for the argument of the logarithm to be positive, only some values of $C$ are possible: $1-C-v>0$ i.e. $C<0.15$. The conservation of matter requires that for a space period of span $\frac{2 \pi}{K}$, the total volume should be equal to $\int_{-\pi / K}^{\pi / K} h_{\mathrm{SAT}}(x) \mathrm{d} x=\frac{2 \pi}{K} h_{0}$. This relationship is compatible with the range of allowed values for $C$ only if the initial thickness of the film is inferior to $70 \mathrm{~nm}$.

However, most of our experiments were carried out on layers much thicker than $70 \mathrm{~nm}$ and saturation was observed for all of them. This was also broadly reported by other groups. This means that the diffusion model in itself, without any bleaching process or another phenomenon coming into play, cannot account for the saturation that is observed. Saturation could be viewed 
as a consequence of the bleaching process. If we stick to the conservation equation written above, it appears that a smaller absorption coefficient $\beta$ contained in $h_{\mathrm{SAT}}(x)$ would guarantee compatibility between the conservation of matter and the expression of $h_{\mathrm{SAT}}(x)$ for much bigger values of $h_{0}$. In other words, the partial loss of optical activity of the layer favours the balance of the diffusion process and indirectly could lead to saturation.

Of course, this expression of the saturation value drawn above and used here is not valid anymore for an absorption coefficient $\beta$ that varies in time but it gives a hint as to how the coupling may work. In addition to that point, the simplistic assumption that the characteristic optical activity lifetime $\tau$ is uniform all over the free surface of the sample amounts to underestimating the effect of the bleaching process in time and its coupling to the self-limitation of the diffusion process. Indeed, given that the most illuminated areas-where molecules are more likely to be activated - are also those which are subjected to maximal bleaching effects, the balancing of the flux between brighter, more depleted areas and darker, more populated areas is enhanced by the bleaching process (figure 13).

3.2.3. The model also accounts for the effects of a polarization modulated pattern. As stressed in section 1.1.3, a light pattern of uniform intensity with periodically modulated polarization direction gives a surface relief grating that is similar to those observed when the light intensity is modulated. The diffusion model presented in section 2 accounts for this observation. Let us assume that the intensity is uniform and that the linear polarization direction rotates periodically along the $(O x)$ axis. Light intensity being uniform, the isomerization probability, thus the probability that a chromophore is put in motion, is the same at a given $z$ all over the sample.

However, the motion of the azobenzene chromophore is assumed to occur along the polarization direction. This way, the direction of the motion also varies periodically along the $x$ axis. On figure 14, we can qualitatively grasp that the effective motion which actually leads to the building of the grating is the one that occurs along the $x$ axis. A quantitative approach based an effective modulated mean free path $l_{x}(x)$ along the $(O x)$ direction injected in the evolution equation leads to the result that we observed experimentally.

\section{Characteristics of the light-induced molecular motion}

\subsection{Estimation of the random-walk step}

As stressed in section 1, observing the light-induced motion of isolated molecules is quite tricky. This is why, based on the model developed above, we propose an estimation of the step of the random-walk performed by the chromophore and the distance it crosses during its optical activity lifetime. Equating the theoretical initial growth rate $\mathrm{d} h_{1}(0) / \mathrm{d} t$ that we obtained in section 3.2.1 to the experimental value leads to an order of magnitude for the step $l$ of the assumed random-walk. This result is of major importance in the context of our model: is the value of $l$ obviously nonsensical so that it overrules the model? If not, does it leave hope of observing a single molecule motion?

Let us consider a $275 \mathrm{~nm}$ thick PMMA DR1 layer, subjected to an interference pattern with a $0.85 \mu \mathrm{m}$ periodicity and a polarization direction perpendicular to the fringes. In these experimental conditions, the grating growth evolution versus time displays an initial grating growth rate of $1 \mathrm{~nm} \mathrm{~s}^{-1}$.

In section 3.2.1, we computed the following expression for the initial grating growth:

$$
\frac{\mathrm{d} h_{1}}{\mathrm{~d} t}(0)=-K^{2} D v A \text {. }
$$

In the experimental conditions mentioned above, we can estimate the values of $v, K$ and $A$. 


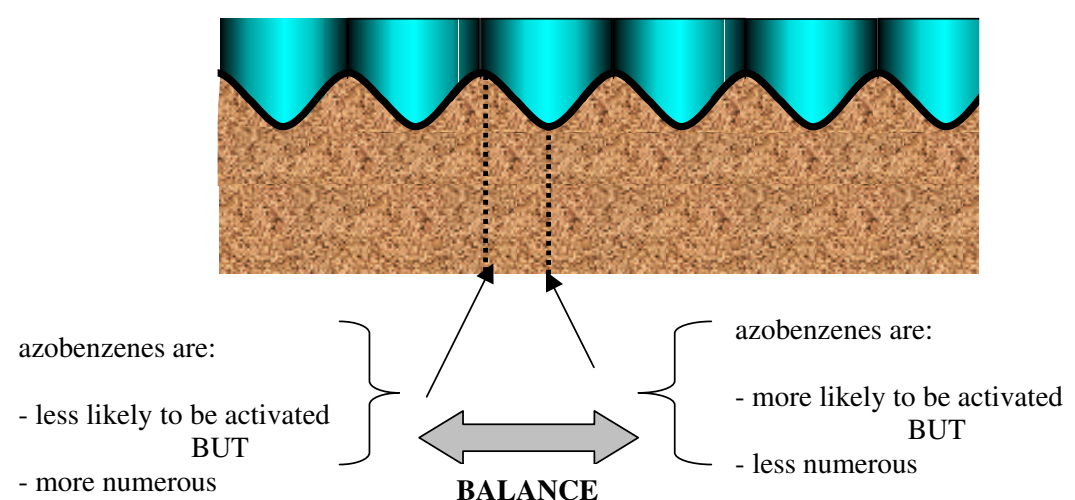

Figure 13. Interpretation of the phenomenon of saturation as the result of the combination of the self-limited diffusion in the film enhanced by the bleaching process.

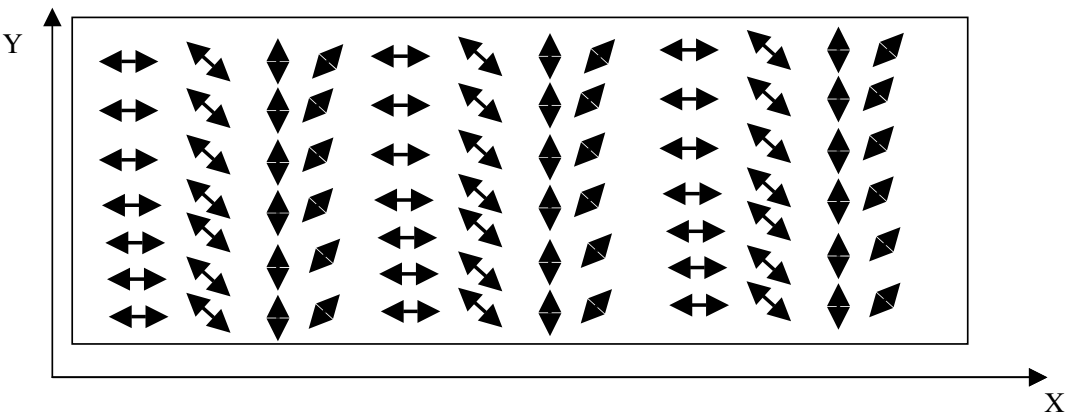

Figure 14. Two counter-propagating waves result in a profile of uniform intensity and modulated polarization direction.

- The $\Lambda=0.85 \mu \mathrm{m}$ fringe spacing is obtained for a $32^{\circ}$ angle between the interfering beams.

- In a p polarization configuration, the visibility factor is $v=\cos (32)=0.85$.

- Concerning the wavenumber $K=2 \pi / \Lambda$, we get $K=7.5 \times 10^{6} \mathrm{~m}^{-1}$.

- As for the absorption coefficient, the initial absorption resulting from the fast optical activity decrease is $A=0.75$. This leads to $D \approx 2.5 \times 10^{-23} \mathrm{~m}^{3} \mathrm{~s}^{-3}$. As defined above, $D=l^{2} \phi \frac{\lambda}{h c} I_{0} V$, with $\lambda=473 \mathrm{~nm}, h c \approx 2 \times 10^{-25} \mathrm{~kg} \mathrm{~m}^{3} \mathrm{~s}^{-2}, I_{0}=1500 \mathrm{~W} \mathrm{~m}^{-2}$.

Following the second assumption (A2), the volume $V$ which the chromophore drags is the PMMA DR1 elementary statistical unit to which an azobenzene is grafted. We assess the volume $V$ of this elementary unit to be around $1 \mathrm{~nm}^{3}$. Thus, we can deduce for the randomwalk step $l \approx 1-2 \mathrm{~nm}$. At first sight, such a value for the mean free path in polymers may seem quite large. However, the motion of polymers drastically differs when the solicitation is small compared to the typical intricacy distance. Nevertheless, the order of magnitude found for $l$ is small compared to the light pattern modulation, which is around $1 \mu \mathrm{m}$. This brings an $a$ posteriori justification to the first-order development done above. We shall now use this value of $l$ to discuss the possibility of experimentally observing the motion of an isolated molecule or a group of isolated molecules. 


\subsection{Distance covered by a chromophore before bleaching}

In this section, we assess the distance covered by an azobenzene chromophore before it is bleached. Let us consider a chromophore deposited on a surface with which it can interact. The chromophore is located in the field of a linearly polarized laser whose intensity is equal to $15 \mathrm{~mW} / 10 \mathrm{~mm}^{2}$. The polarization direction is collinear to the surface on which the chromophore is deposited.

- In the framework of the model presented above each step $l$ performed by the chromophore is around a $1-2 \mathrm{~nm}$.

- The rate at which a molecule is shifted by a step $l$ can be found out by dividing the number of isomerized molecules by unit volume and by unit time $n_{\mathrm{d}}$ by the density of molecules.

The number of molecules per unit volume and per unit time $n_{\mathrm{d}}$ which undergo diffusion motion is

$$
n_{\mathrm{d}}=\phi \beta \frac{\lambda}{h c} I
$$

With the values of the parameters already given above, we calculate $n_{\mathrm{d}} \approx 5 \times 10^{17}$ azobenzene functions moving every second per cubic millimetre. Besides, using the density of PMMA DR1 $(1,1)$ and given that a statistical unit of PMMA DR1 consists in $30 \%$ of DR1 and $70 \%$ of PMMA, we calculate that one cubic millimetre contains $10^{18}$ azobenzene functions. Since we estimated that around $n_{\mathrm{d}}=5 \times 10^{17}$ azobenzene functions are put in motion every second per cubic millimetre, we can estimate that each azobenzene chromophore undergoes diffusion motion every two seconds. So, after $t$ seconds, the azobenzene chromophore has undergone $N=\frac{t}{2}$ random-walk movements. If we follow assumption (A3), the azobenzene chromophores perform a one-dimensional random walk of mean free path $l$ along the polarization direction. Thus, after $N$ shifts, the azobenzene function has covered a distance equal to $\sqrt{N l}$, typical of a random walk. This means that, at time $t$, the distance covered by the chromophore is $\sqrt{\frac{t}{2}} l$. For a $15 \mathrm{~mW} / 10 \mathrm{~mm}^{2}$ intensity linearly polarized laser beam, we have estimated the lifetime activity to be around $40 \mathrm{~min}$, that is to say $T=2400 \mathrm{~s}$, as reported in section 1.3 . From the value of $l$ computed in section 4.1 and the evaluation of the distance crossed $t$ seconds after the beginning of the exposure, we can assess the distance covered by the azo-chromophore during its optical activity lifetime, considered in that example to be $T=2400 \mathrm{~s}$ :

distance covered during the optical activity lifetime $=\sqrt{\frac{T}{2}} l \approx 100 \mathrm{~nm}$.

In this framework, the motion of a single molecule before bleaching should be easily observed with an AFM, provided that the interaction with the surface is controlled.

\subsection{Energetic output of the light-driven random walk}

In our model, one photon of energy $4 \times 10^{-19} \mathrm{~J}$ causes a PMMA DR1 unit to move of several nanometres, the mass of one unit being estimated to be equivalent to 61 carbon atoms, that is to say $1.2 \times 10^{-21} \mathrm{~g}$. The energy input per unit mass is $330 \mathrm{~J} \mathrm{~g}^{-1}$. This can be compared to the conversion of the energy contained in ATP (adenosine triphosphate) into the motion of a kinesin molecule along a microtubule, which is the molecular motion on which are based muscular processes [12]. In this case, a molecule of kinesin of mass $6.5 \times 10^{-19} \mathrm{~g}$ performs an eight-nanometre step using the energy contained in one molecule of ATP, which is equal to $5 \times 10^{-20} \mathrm{~J}$. The energy input per unit mass is $0.08 \mathrm{~J} \mathrm{~g}^{-1}$. This shows that, although noteworthy, the light-dragged mass motion is much less efficient than the kinesin motion. 


\section{Discussion over the microscopic phenomenon at work}

It is somewhat tempting to consider the hypotheses formulated in section 2.1 in the light of the theory of 'thermal ratchets' introduced by Feynman in the mid-1960s and later developed by Magnasco [13] as well as by Prost et al [14]. In the framework of this theory, a particle is submitted alternatively to two periodic and asymmetrical potentials of periodicity $L$, or equivalently a particle alternates between two states, thus alternatively undergoing two periodic and asymmetrical potentials.

The possibility of a motion lies on the existence of a space asymmetry of the periodic potential to which each state is subjected. It also requires a time symmetry breaking. Ratchets are systems that combine asymmetry with non-equilibrium processes to generate directed particle flow. This latter is introduced by an optical or a chemical pumping which breaks the balance between the occupation probabilities of the two states. In this framework, thermal fluctuations are turned into work. The model of thermal ratchets describes adequately the behaviour of biological molecular motors such as kinesin. It also shares points in common with the light-driven motion presented in this paper.

Indeed, in both cases the shifting of a particle results from two distinct motions at two different scales: a short distance random motion and a directed motion driven by a long distance space asymmetry. The short range random motion, associated to the isomerization in assumption (A3), is of thermal origin in the thermal ratchet model. As for the long range space asymmetry, which is caused by the light intensity or polarization modulation in our assumptions, it is due to the shape of the periodic potential undergone by the thermal ratchet. Moreover, the motion requires transitions between two states and an energy supply which breaks the population rate of the two levels. However, this model does not provide an altogether suitable description for the light-induced motion of azobenzene-containing materials. A major difference lies in the nature of the small scale motion. As opposed to thermal ratchets, where the small scale motion is the continual thermal agitation, it is only under isomerization that azobenzenes move randomly. Moreover, it is at this point that azobenzenes perform a step whereas thermal ratchets' steps occur in a directed way. For a thermal ratchet, the load is driven directly by thermal fluctuations and its diffusive motion is rectified by the energy intake and the asymmetric potential profile. As for the motion here, it is a non-corrected random walk, that is to say a much less directional motion. Moreover, there is no physical reason why the interaction potential between the chromophore dipole and the electromagnetic field should be asymmetrical. Even if it were so, it would lead to a micrometric and thus outright nonsensical value for the mean free path $l$. Indeed, the periodicity of the interaction potential should be that of the electric field, that is to say around $1 \mu \mathrm{m}$. The associated 'thermal ratchet' motion should have the same periodicity, which means that the azobenzene chromophore's step should be around $1 \mu \mathrm{m}$. Nevertheless, we shall retain from this model the necessity of both a space and a time symmetry breaking. Indeed, the motion requires an interaction with its environment, the substrate for instance, that is to say a dissipative process which breaks the time symmetry.

Before concluding, it is important to mention that a model for light-induced motion of individual molecules, which include chromophores, was introduced in 2000 by Porto et al [15]. This model proposes basic principles for an engine transforming energy into directed translational or rotational motion at the nanoscale. The approach that we suggest in our paper shares some aspects with the model developed by Porto et al. Indeed, it does not require a space asymmetry neither of the moving object nor of the interaction potential with the surface. Moreover, the moving object is, in both cases, likely to drag an inactive cargo. However, this model does not altogether apply to our experimental conditions for several reasons. First, quoting [15]: 'the approach is based on the transformation of the fed energy to directed motion 
through a dynamical competition between the intrinsic lengths of the moving object and the supporting carrier'. An essential point in Porto's model is that the object is made up of $N$ interacting particles linked by bounds whereas in the case of Frechet's moving molecules depicted in figure 6 , there is only one active element per moving molecule. Thus, in our model, there is no concept of intrinsic lengths of the moving object. As for the reference made by Porto et al to the use of chromophores, it also does not apply to the molecules that we used since in their model the photochromic molecules play the role of bonds controlled by lightinduced conformational changes whereas the chromophores are located at the periphery (for POPAM dendrimers), in the side chains (for PMMA DR1) or as a single peripheral moiety (for Frechet's molecules). Besides, in Porto's model, the extension of the molecule, given by the minimum rest length, is of the same order of magnitude as the period of the space potential, whereas in the protocol presented in this paper the period of the intensity pattern which defines the space modulation is $1 \mu \mathrm{m}$, that is to say around one thousand larger than the extension of the moving object. Finally, if the notion of a space-correlated excitation is common to both cases, the notion of a time-correlated excitation is not an element of our model, the time coherence of the laser being important only for the interference pattern to be large enough.

\section{Conclusion and perspectives}

The random-walk model discussed here accounts for the light-induced mass motion observed in azobenzene-containing thin films. The value of the step $l$ associated to the random walk found here, $1-2 \mathrm{~nm}$, is quite large. In the context of this model, the bleaching process appears to play a major limiting role. One way to check that assumption would be to work in a bleachinglimiting environment. Working in a high vacuum environment could be a solution, unless the oxidant agents are included in the azobenzene-containing molecule itself. In this case, working at a lower temperature could be an alternative.

\section{Acknowledgments}

This work has been achieved through a European collaboration: the LIMM (Light Induced Molecular Motion) project financed by the European Union under contract number IST-200135503.

We would like to thank all our partners involved in this collaboration and especially Professor F Vögtle and his group from the Kekulé-Institut für Organische Chemie und Biochemie in Bonn, who provided us with the azobenzene dendrimers which have proved quite helpful in the development of this work.

\section{References}

[1] Rochon P, Batalla E and Natansohn A 1995 Appl. Phys. Lett. 66136

[2] Pedersen T G, Johansen P M, Holme N C R and Ramanujam P S 1998 Phys. Rev. Lett. 8089

[3] Bublitz D, Helgert M, Fleck B, Wenke L, Hvilsted S and Ramanujam P S 2000 Appl. Phys. B 70863

[4] Kumar J, Li L, Liang X L, Kim D Y, Lee T S and Tripathy S K 1998 Appl. Phys. Lett. 722096

[5] Viswanathan N K, Balasubramanian S, Li L, Kumar J and Tripathy S K 1998 J. Phys. Chem. B 722096

[6] Baldus O and Zilker S 2001 Appl. Phys. B 72425

[7] Delaire J A and Nakatani K 2002 Chem. Rev. 1001817

[8] Natansohn A and Rochon P 2003 Chem. Rev. 1024139

[9] Archut A, Vögtle F, De Cola L, Azzellini G C, Balzani V, Ramanujam P S and Berg R H 1998 Chem. Eur. J. 4699

[10] Rau H 1990 Photochemestry of azobenzenes Photochemestry and Photophysics vol 2, ed J Rebek (Boca Raton, FL: CRC Press) p 119

[11] Ackermann J, Bellini B, Klein H, Dumas Ph and Safarov V 2006 in preparation

[12] http://www.proweb.org/kinesin/KinesinMotility.html

[13] Magnasco M 1993 Phys. Rev.Lett. 711477

[14] Prost J, Chauwin J F, Peliti L and Adjari A 1994 Phys. Rev.Lett. 722652

[15] Porto M et al 2000 Phys. Rev.Lett. 846058 